\title{
ESTUDO FITOSSOCIOLÓGICO DE UMA COMUNIDADE VEGETAL SOBRE CANGA COMO SUBSÍdIO PARA A REABILITAÇÃO DE ÁREAS MINERADAS NO QUADRILÁTERO FERRÍFERO, MG ${ }^{1}$
}

\author{
Claudia Maria Jacobi², Flávio Fonseca do Carmo ${ }^{3}$ e Regina de Castro Vincent ${ }^{4}$
}

\begin{abstract}
RESUMO - O objetivo deste trabalho foi caracterizar a estrutura e composição de um campo rupestre sobre canga para servir de base a estudos sobre reabilitação de áreas degradadas pela mineração de ferro. Estudouse uma canga no Parque Estadual da Serra do Rola-Moça, MG. Em 30 parcelas de $2 \mathrm{~m}^{2}$, foram amostrados 2.151 indivíduos pertencentes a 32 espécies e 16 famílias, com diversidade de 2,45 nats/ind. A altura média foi de 15,8 $\pm 16,3 \mathrm{~cm}$, com $80 \%$ dos indivíduos menores do que $25 \mathrm{~cm}$. As famílias mais importantes foram Orchidaceae, Poaceae e Cyperaceae, e as espécies com maior valor de importância foram Andropogon ingratus (Poaceae), Lychnophora pinaster (Asteraceae), Bulbostylis fimbriata (Cyperaceae), Sophronitis caulescens (Orchidaceae) e Sebastiania glandulosa (Euphorbiaceae). Sugere-se que essas espécies mais importantes, aquelas com crescimento clonal como gramíneas, ciperáceas e orquídeas epilíticas, as facilitadoras como Stachytarpheta glabra e Mimosa calodendron e espécies tolerantes a metais pesados como Vellozia spp. sejam candidatas prioritárias em programas de recuperação de áreas degradadas por mineração de ferro.
\end{abstract}

Palavras-chave: Campos rupestres, fitossociologia e espécies facilitadoras.

\section{PHYTOSOCIOLOGICAL STUDY OF A PLANT COMMUNITY ON IRONSTONE AS SUPPORT FOR RECOVERY OF A MINED AREA IN THE IRON QUADRANGLE, MG}

\begin{abstract}
This work aimed to characterize the structure and composition of a Rupestrian field over ironstone as a basis for rehabilitation studies of areas degraded by iron mining activities. An ironstone outcrop at Serra do Rola Moça State Park, MG, was studied. In 30 plots of $2 \mathrm{~m}^{2}, 2,151$ individuals were found, belonging to 32 species and 16 families, with a diversity of 2.45 nats/ind. Mean height was $15.7 \pm 16.3 \mathrm{~cm}$, with $80 \%$ below $25 \mathrm{~cm}$. The most important families were Orchidaceae, Poaceae, and Cyperaceae, and the species with highest importance value were Andropogon ingratus (Poaceae), Lychnophora pinaster (Asteraceae), Bulbostylis fimbriata (Cyperaceae), Sophronitis caulescens (Orchidaceae), and Sebastiania glandulosa (Euphorbiaceae). We suggest that these species, together with those presenting clonal growth, such as grasses, sedges and epilythic orchids; facilitators, such as Stachytarpheta glabra and Mimosa calodendron; and those tolerant to heavy metals, such as Vellozia spp., be priority candidates in restoration programs in areas degraded by iron ore extraction.
\end{abstract}

Keywords: Rupestrian fields, phytosociology and facilitator species.

\footnotetext{
${ }^{1}$ Recebido em 30.01.2007 e aceito para publicação em 20.02.2008.

${ }^{2}$ Departamento de Biologia Geral da Universidade Federal de Minas Gerais (UFMG). E-mail: <jacobi@ mono.icb.ufmg.br>.

${ }^{3}$ Programa de Pós-Graduação em Ecologia, Conservação e Manejo da Fauna Silvestre da UFMG. E-mail: <flaviofc@ufmg.br>.

${ }^{4}$ Departamento de Avaliação de Impacto Ambiental (DAIA-SMA/SP). E-mail: <regina_c_vincent@ yahoo.com.br>.
} 


\section{INTRODUÇÃO}

O Quadrilátero Ferrífero (QF) é uma das mais importantes províncias minerais do mundo (SPIER et al., 2003). O Brasil é o segundo produtor mundial de minério de ferro, sendo cerca de $70 \%$ extraído nessa região. Atualmente, existem ali mais de 50 minas a céu aberto em atividade (DNPM, 2006), resultando na completa alteração da paisagem, com enormes impactos nas biodiversidades local e regional (BRADSHAW, 1997; TOY e GRIFFITH, 2001; TEIXEIRA e LEMOS FILHO, 2002).

Os campos rupestres ferruginosos, conhecidos como vegetação de canga, com distribuição em áreas restritas associadas a importantes depósitos de minério de ferro, estão entre os ecossistemas mais ameaçados e menos estudados de Minas Gerais (JACOBI et al., 2007; JACOBI e CARMO, 2008a), abrigando espécies vegetais com potencial medicinal como a "arnica" (Lychnophora pinaster Mart.), ornamental como bromélias e orquídeas e plantas tolerantes a altas concentrações de metais pesados no substrato (PORTO e SILVA, 1989; RIZZINI, 1997; TEIXEIRA e LEMOS FILHO, 1998), conhecidas como metalófilas.

Os programas de recuperação de áreas degradadas devem ser concomitantes com todas as etapas de mineração, não se restringindo ao término da exploração (BARTH, 1989). Entretanto, segundo Toy et al. (2001) as empresas de mineração no Brasil continuam com práticas de recuperação de curto prazo, apesar da criação, em 1989, da exigência de um Plano para Recuperação de Áreas Degradadas (PRAD) durante processos de licenciamento ambiental (Decreto $n^{\circ}$ 97.632/89, Art. $1^{\circ}$ ). Atualmente, os órgãos responsáveis pela regulamentação das minas de superfície começaram a priorizar metas de longo prazo, incluindo o uso futuro da terra (TOY et al., 2001). Isso constitui um avanço importante para integrar ao ecossistema áreas com suas funções ecológicas recuperadas, permitindo o desenvolvimento econômico com menor passivo ambiental.

Uma relevante lacuna nesses programas diz respeito ao resgate e à recuperação dos campos rupestres, que ainda não possuem legislação específica para a sua conservação e utilização (MEIRELLES et al., 1999). As comunidades rupestres associadas a substratos metalíferos, apesar de sua inestimável importância ambiental, ainda são negligenciadas, resultando no desconhecimento da dinâmica desses ecossistemas e do potencial da vegetação nativa para o uso sustentável ou para a recuperação de áreas degradadas por mineração (GINOCCHIO e BAKER, 2004).

Uma das estratégias mais recomendadas em planos de recuperação é reproduzir o padrão natural das comunidades vegetais, o que aumenta a probabilidade de sucesso na recuperação ambiental, além de contribuir para reduzir os custos desses projetos (ARAÚJO et al., 2006). Estudos fitossociológicos são fundamentais para o conhecimento desses padrões, abrangendo os fenômenos que afetam a dinâmica dessas comunidades, sua constituição, classificação, proporção na abundância entre espécies e distribuição espacial dos indivíduos (MUELLER-DOMBOIS e ELLENBERG, 1974).

Este trabalho teve como objetivo caracterizar uma comunidade vegetal de afloramento ferruginoso quanto à sua estrutura fitossociológica e utilizar os dados obtidos, para indicar espécies nativas com potencial para a utilização em programas de reabilitação de áreas degradadas pela mineração de ferro.

\section{MATERIAL E MÉTODOS}

O estudo foi realizado no Parque Estadual da Serra do Rola Moça-PESRM (2003'60'S, 4402'00''W; $c a$. $1.450 \mathrm{~m}$ de altitude), noroeste do Quadrilátero Ferrífero, que integra a Área de Proteção Ambiental (APA) Sul RMBH, região prioritária para a conservação da biodiversidade de Minas Gerais (DRUMMOND et al., 2005). O clima da região é do tipo CWb, com verões quentes e chuvosos e invernos secos. A precipitação total anual é de 1.000 a $1.550 \mathrm{~mm}$ e a temperatura média anual, de $18{ }^{\circ} \mathrm{C}$ (IBRAM, 2003).

O parque encontra-se numa região de transição entre os domínios morfoclimáticos de Cerrados e de Floresta Tropical Atlântica senso Ab'Saber (1977), abrigando diversas fitofisionomias, como Cerrado sensu strictu, campo sujo, Floresta Estacional Semidecidual, florestas ciliares e, nos topos das serras, a aproximadamente 1.200-1.500 m de altitude, os campos rupestres ferruginosos, desenvolvendo-se sobre canga. A canga, termo brasileiro para um depósito ferruginoso superficial composto por minerais derivados de formações ferríferas bandadas (SIMMONS, 1963), contém um mosaico de ambientes, entre os quais se destacam a superfície rochosa, fendas e depressões, capões, pequenos lagos temporários e cavernas (JACOBI et al., 2007). 
O levantamento fitossociológico foi realizado nos ambientes formados por rocha exposta, fendas e depressões, por sua maior representatividade espacial e fisionômica local, uma vez que essas formações representam cerca de $90 \%$ da área total da canga estudada (aproximadamente 20 ha). Foi delimitada uma área de $2.500 \mathrm{~m}^{2}$, formada por cinco faixas de $10 \mathrm{~m} \times 50 \mathrm{~m}$. Em cada faixa, foram sorteadas seis parcelas de $2 \mathrm{~m} \times 1 \mathrm{~m}$, totalizando 30 parcelas.

Em cada parcela, foram anotadas a espécie, a área de cobertura e a altura de cada indivíduo. Foram considerados todos os indivíduos acima de $1 \mathrm{~cm} \mathrm{de}$ altura para as espécies herbáceas e acima de $5 \mathrm{~cm}$ para as lenhosas. No caso das espécies com reprodução clonal (orquídeas e gramíneas, entre outras), cada touceira isolada foi considerada como um indivíduo. A partir desses dados, foram calculados os parâmetros fitossociológicos: densidade, freqüência e dominâncias absoluta e relativa e os índices de valor de importância (IVI) e de cobertura (IVC), além da diversidade e equabilidade (índices de Shannon-Wiener e Pielou) (MUELLER-DOMBOIS e ELLENBERG, 1974). A porcentagem de substrato exposto foi obtida utilizandose uma grade com área interna de $0,5 \mathrm{~m} \times 0,5 \mathrm{~m}$, dividida a intervalos de $10 \mathrm{~cm}$ por fios de náilon. Material testemunho foi depositado no herbário BHCB, da Universidade Federal de Minas Gerais.

\section{RESULTADOS E DISCUSSÃO}

Foram amostrados 2.151 indivíduos, distribuídos em 32 espécies e 16 famílias, com densidade total de cerca de 36 ind. $/ \mathrm{m}^{2}$ (Quadro 1). O valor da diversidade foi de 2,45 nats/ind. e o de equabilidade, 0,71 , refletindo o predomínio de indivíduos de Bulbostylis fimbriata C. B. Clarke (Cyperaceae), Andropogon ingratus Hack. (Poaceae) e Sophronitis caulescens (Lindl.) Van den Berg \& M. W. Chase (Orchidaceae) e a baixa densidade (menos de cinco indivíduos) de 10 espécies. As comunidades vegetais sobre afloramentos rochosos geralmente apresentam baixa diversidade de espécies, em comparação com ecossistemas do seu entorno (RIZZINI, 1997), tendo sido encontrados valores de 2,79 nats/ind. em um afloramento quartzítico da Chapada Diamantina, BA (CONCEIÇÃO e GIULIETTI, 2002), 2,53 nats/ind. em afloramento de canga na Serra da Mutuca, QF (VINCENT, 2004) e de 1,74 a 2,96 nats/ind. em afloramentos graníticos no Rio de Janeiro (MEIRELLES et al., 1999). No entanto, recentes estudos florísticos em campos rupestres sobre canga (VIANA e LOMBARDI, 2007; JACOBI e CARMO, 2008b) evidenciam que esses campos sejam mais diversos do que o imaginado.

Quadro 1 - Parâmetros fitossociológicos das espécies amostradas em canga, Parque Estadual Serra do Rola Moça, MG. N: número de indivíduos; DA: densidade absoluta; DR: densidade relativa; DoA: dominância absoluta; DoR: dominância relativa; FA: freqüência absoluta; FR: frequiência relativa; IVC: índice de valor de cobertura; e IVI: índice de valor de importância

Table 1 - Phyto-sociological parameters of the species sampled in an ironstone outcrop, Serra do Rola Moça State Park, MG, Brazil. N: number of individuals; DA: absolute density; DR: relative density; DoA: absolute dominance; DoR: relative dominance; FA: absolute frequency; FR: relative frequency; IVC: cover value index; IVI: importance value index

\begin{tabular}{|c|c|c|c|c|c|c|c|c|c|c|}
\hline Espécie & Família & $\mathrm{N}$ & $\begin{array}{c}\mathrm{DA} \\
\left.\text { (ind } / \mathrm{m}^{2}\right)\end{array}$ & $\begin{array}{c}\mathrm{DR} \\
\%\end{array}$ & $\begin{array}{c}\operatorname{DoA} \\
\left(\mathrm{cm}^{2} / \mathrm{m}^{2}\right)\end{array}$ & $\begin{array}{c}\text { DoR } \\
\%\end{array}$ & FA & $\begin{array}{c}\mathrm{FR} \\
\%\end{array}$ & IVC & IVI \\
\hline Andropogon ingratus Hack. & Poaceae & 400 & 6,67 & 18,60 & 917,37 & 8,75 & 90,00 & 9,44 & 13,67 & 12,26 \\
\hline Lychnophora pinaster Mart. & Asteraceaee & 51 & 0,85 & 2,37 & 2341,59 & 22,32 & 70,00 & 7,34 & 12,35 & 10,68 \\
\hline Bulbostylis fimbriata C.B.Clarke & Cyperaceae & 414 & 6,90 & 19,25 & 215,22 & 2,05 & 73,33 & 7,69 & 10,65 & 9,66 \\
\hline Sophronitis caulescens (Lindl.) & Orchidaceae & 328 & 5,47 & 15,25 & 495,44 & 4,72 & 73,33 & 7,69 & 9,99 & 9,22 \\
\hline Van den Berg \& M.W. Chase & & & & & & & & & & \\
\hline Sebastiania glandulosa (Mart.) Pax & Euphorbiaceae & 152 & 2,53 & 7,07 & 796,60 & 7,59 & 86,67 & 9,09 & 7,33 & 7,92 \\
\hline Stachytarpheta glabra Cham. & Verbenaceae & 65 & 1,08 & 3,02 & 1363,98 & 13,00 & 60,00 & 6,29 & 8,01 & 7,44 \\
\hline $\begin{array}{l}\text { Symphyopappus brasiliensis } \\
\text { (Gardner) R.M. King \& H. Rob. }\end{array}$ & Asteraceae & 114 & 1,90 & 5,30 & 603,87 & 5,76 & 80,00 & 8,39 & 5,53 & 6,48 \\
\hline Acianthera teres (Lindl.) Borba & Orchidaceae & 222 & 3,70 & 10,32 & 127,21 & 1,21 & 36,67 & 3,85 & 5,77 & 5,13 \\
\hline Paspalum scalare Trin. & Poaceae & 90 & 1,50 & 4,18 & 320,97 & 3,06 & 50,00 & 5,24 & 3,62 & 4,16 \\
\hline Vellozia graminea Pohl & Velloziaceae & 95 & 1,58 & 4,42 & 260,14 & 2,48 & 50,00 & 5,24 & 3,45 & 4,05 \\
\hline
\end{tabular}

R. Árvore, Viçosa-MG, v.32, n.2, p.345-353, 2008 
Tabela 1 - Cont.

Table 1 - Cont

\begin{tabular}{|c|c|c|c|c|c|c|c|c|c|c|}
\hline Espécie & Família & $\mathrm{N}$ & $\begin{array}{c}\mathrm{DA} \\
\left(\mathrm{ind} / \mathrm{m}^{2}\right) \\
\end{array}$ & $\begin{array}{c}\mathrm{DR} \\
\% \\
\end{array}$ & $\begin{array}{c}\mathrm{DoA} \\
\left(\mathrm{cm}^{2} / \mathrm{m}^{2}\right)\end{array}$ & $\begin{array}{c}\mathrm{DoR} \\
\% \\
\end{array}$ & FA & $\begin{array}{c}\text { FR } \\
\%\end{array}$ & IVC & IVI \\
\hline Tibouchina multiflora Cogn. & Melastomataceae & 24 & 0,40 & 1,12 & 543,96 & 5,19 & 46,67 & 4,90 & 3,15 & 3,73 \\
\hline Vellozia compacta Mart. & Velloziaceae & 61 & 1,02 & 2,84 & 569,46 & 5,43 & 26,67 & 2,80 & 4,13 & 3,69 \\
\hline Struthanthus flexicaulis Mart. & Loranthaceae & 5 & 0,08 & 0,23 & 847,35 & 8,08 & 16,67 & 1,75 & 4,16 & 3,35 \\
\hline Mimosa calodendron Mart. & Fabaceae & 10 & 0,17 & 0,46 & 247,43 & 2,36 & 20,00 & 2,10 & 1,41 & 1,64 \\
\hline $\begin{array}{l}\text { Arthrocereus glaziovii (K. Schum.) } \\
\text { N.P.Taylor \& D.C. Zappi }\end{array}$ & \multicolumn{9}{|c|}{ N.P.Taylor \& D.C. Zappi } & 1,46 \\
\hline Baccharis serrulata Pers. & Asteraceae & 16 & 0,27 & 0,74 & 166,01 & 1,58 & 16,67 & 1,75 & 1,16 & 1,36 \\
\hline Lagenocarpus rigidus Nees & Cyperaceae & 8 & 0,13 & 0,37 & 314,58 & 3,00 & 6,67 & 0,70 & 1,69 & 1,36 \\
\hline Portulaca hirsutissima Cambess. & Portulacaceae & 14 & 0,23 & 0,65 & 0,79 & 0,01 & 26,67 & 2,80 & 0,33 & 1,15 \\
\hline Ditassa mисronata Mart. & Apocynaceae & 14 & 0,23 & 0,65 & 20,82 & 0,20 & 20,00 & 2,10 & 0,42 & 0,98 \\
\hline Epidendrum secundum Vell. & Orchidaceae & 20 & 0,33 & 0,93 & 45,06 & 0,43 & 6,67 & 0,70 & 0,68 & 0,69 \\
\hline Eriope macrostachya Mart. ex Benth. & Lamiaceae & 4 & 0,07 & 0,19 & 33,35 & 0,32 & 13,33 & 1,40 & 0,25 & 0,63 \\
\hline Baccharis reticularia DC. & Asteraceae & 1 & 0,02 & 0,05 & 157,81 & 1,50 & 3,33 & 0,35 & 0,78 & 0,63 \\
\hline Galianthe sp. & Rubiaceae & 8 & 0,13 & 0,37 & 5,96 & 0,06 & 10,00 & 1,05 & 0,21 & 0,49 \\
\hline Chromolaena sp. & Asteraceae & 3 & 0,05 & 0,14 & 10,88 & 0,10 & 6,67 & 0,70 & 0,12 & 0,31 \\
\hline Periandra mediterranea (Vell.) Taub. & Fabaceae & 3 & 0,05 & 0,14 & 3,13 & 0,03 & 6,67 & 0,70 & 0,08 & 0,29 \\
\hline Axonopus siccus Kuhlm. & Poaceae & 6 & 0,10 & 0,28 & 21,39 & 0,20 & 3,33 & 0,35 & 0,24 & 0,28 \\
\hline Heteropteris sp. & Malpighiaceae & 2 & 0,03 & 0,09 & 14,78 & 0,14 & 3,33 & 0,35 & 0,12 & 0,19 \\
\hline Tibouchina sp. & Melastomataceae & e 1 & 0,02 & 0,05 & 11,03 & 0,11 & 3,33 & 0,35 & 0,08 & 0,17 \\
\hline Trichogonia sp. & Asteraceae & 1 & 0,02 & 0,05 & 6,00 & 0,06 & 3,33 & 0,35 & 0,05 & 0,15 \\
\hline Croton serratoideus & Euphorbiaceae & 2 & 0,03 & 0,09 & 0,89 & 0,01 & 3,33 & 0,35 & 0,05 & 0,15 \\
\hline \multicolumn{11}{|l|}{ Radcl.-Sm. \& Govaerts } \\
\hline Ditassa linearis Mart. & Apocynaceae & 2 & 0,03 & 0,09 & 0,73 & 0,01 & 3,33 & 0,35 & 0,05 & 0,15 \\
\hline Trixis vauthieri DC. & Asteraceae & 1 & 0,02 & 0,05 & 2,41 & 0,02 & 3,33 & 0,35 & 0,03 & 0,14 \\
\hline Total & & 2151 & 35,85 & 100 & 10489,12 & 100 & 953,33 & 100 & 100 & 100 \\
\hline
\end{tabular}

Outro aspecto importante sobre a diversidade de afloramentos em geral é relacionada à sua alta diversidade $\beta$, atribuída ao isolamento entre unidades (BURKE, 2003). Comparações florísticas entre diferentes afloramentos de um mesmo tipo de substrato geológico têm mostrado baixa similaridade (MEIRELLES et al., 1999; VINCENT, 2004). Jacobi et al. (2007), em levantamentos florísticos de duas cangas localizadas no QF, uma na Serra do Rola Moça e outra na Serra da Moeda, distantes apenas 32 $\mathrm{km}$ entre si, encontraram uma similaridade de plantas vasculares de apenas $27 \%$. A indicação de espécies potenciais de canga para uso em programas de restauração deve, portanto, considerar a análise prévia da área-alvo. Apesar da baixa similaridade entre áreas, algumas espécies são freqüentes em cangas do Quadrilátero Ferrífero, como $S$. caulescens, Acianthera teres (Lindl.) Borba (Orchidaceae), Mimosa calodendron Mart. (Fabaceae), Tibochina multiflora Cogn. (Melastomataceae) e Stachytarpheta glabra Cham. (Verbenaceae), presentes no levantamento.

A vegetação no local de estudo foi caracterizada por um estrato herbáceo quase contínuo, com arbustos e subarbustos esparsos, interrompido em alguns trechos por substrato exposto, que constituiu $29 \%$ da área amostrada. A altura média dos indivíduos foi de 15,8 $\pm 16,3 \mathrm{~cm}$. A estrutura vertical foi definida por uma grande quantidade de indivíduos com alturas inferiores a $10 \mathrm{~cm}$ (cerca de 53\%) e $80 \%$ inferiores a $25 \mathrm{~cm}$ (Figura 1). Esse estrato foi composto principalmente por indivíduos de $B$. fimbriata, $A$. ingratus, $S$. caulescens e A. teres. Apenas $5 \%$ dos indivíduos apresentaram altura igual ou superior a $50 \mathrm{~cm}$, representados, sobretudo, por S. glabra, Baccharis serrulata Pers. (Asteraceae), Epidendrum secundum Vell. (Orchidaceae), Sebastiania glandulosa (Mart.) Pax (Euphorbiaceae) e T. multiflora.

Algumas espécies de maior porte, como as arbustivas $M$. calodendron, L. pinaster e S. glabra, foram freqüentemente encontradas em associação com plântulas de outras espécies, principalmente orquídeas. Essas espécies propiciam sombreamento, acúmulo de matéria orgânica e proteção física contra o vento, amenizando as condições ambientais e favorecendo o recrutamento e estabelecimento de plântulas. Plantas 
com essa função facilitadora na comunidade recebem o nome de plantas-berçário (nurse plants). Pesquisas recentes sobre a utilização de plantas-berçário na restauração de ambientes degradados demonstraram as vantagens desses serviços ecológicos, com efeitos positivos na comunidade, como a otimização da sucessão vegetal (CASTRO et al., 2002; BRUNO et al., 2003; PADILLA e PUGNAIRE, 2006).

Projetos de reabilitação de áreas degradadas devem utilizar uma variedade grande de espécies, e um dos critérios de escolha pode ser a representatividade de suas famílias tanto em número de espécies quanto em abundância de indivíduos, uma vez que essa é uma indicação do sucesso do táxon num ambiente particular. As famílias mais ricas no levantamento foram Asteraceae (sete espécies), Poaceae e Orchidaceae (três espécies cada). Apenas três famílias, Orchidaceae, Poaceae e Cyperaceae, somaram $69 \%$ do total de indivíduos amostrados, representando, com Asteraceae, as famílias mais importantes na comunidade (Quadro 2). Asteraceae, Poaceae e Cyperaceae são famílias comuns no Cerrado, e Orchidaceae tem diversos representantes em campos rupestres (GIULIETTI et al., 1987). Considerando que em alguns casos a reabilitação deve ser feita sobre substratos pedregosos, resultantes do depósito dos rejeitos da exploração do minério de ferro, é necessário incluir também espécies relacionadas aos trechos de substrato onde há maior quantidade de solo, como gramíneas, ciperáceas e asteráceas. Entretanto, em diversos resgates de flora presenciados por esses autores, Poaceae e Cyperaceae são preteridas.

As cinco espécies com maior VI foram $A$. ingratus, Lychnophora pinaster Mart. (Asteraceae), B. fimbriata, S. caulescens e $S$. glandulosa, somando quase $50 \%$ do valor de importância (Quadro 1). A. ingratus, $L$. pinaster e $S$. caulescens também estiveram entre as espécies mais importantes em uma canga localizada na Serra da Mutuca (VINCENT, 2004), distante cerca de $5 \mathrm{~km}$ da canga estudada. Enquanto as espécies herbáceas mais importantes apresentaram elevada abundância, a arbustiva L. pinaster se destacou quanto à biomassa, alcançando 22,3\% de dominância relativa, mas apenas 2,4\% de densidade relativa (Quadro 1).

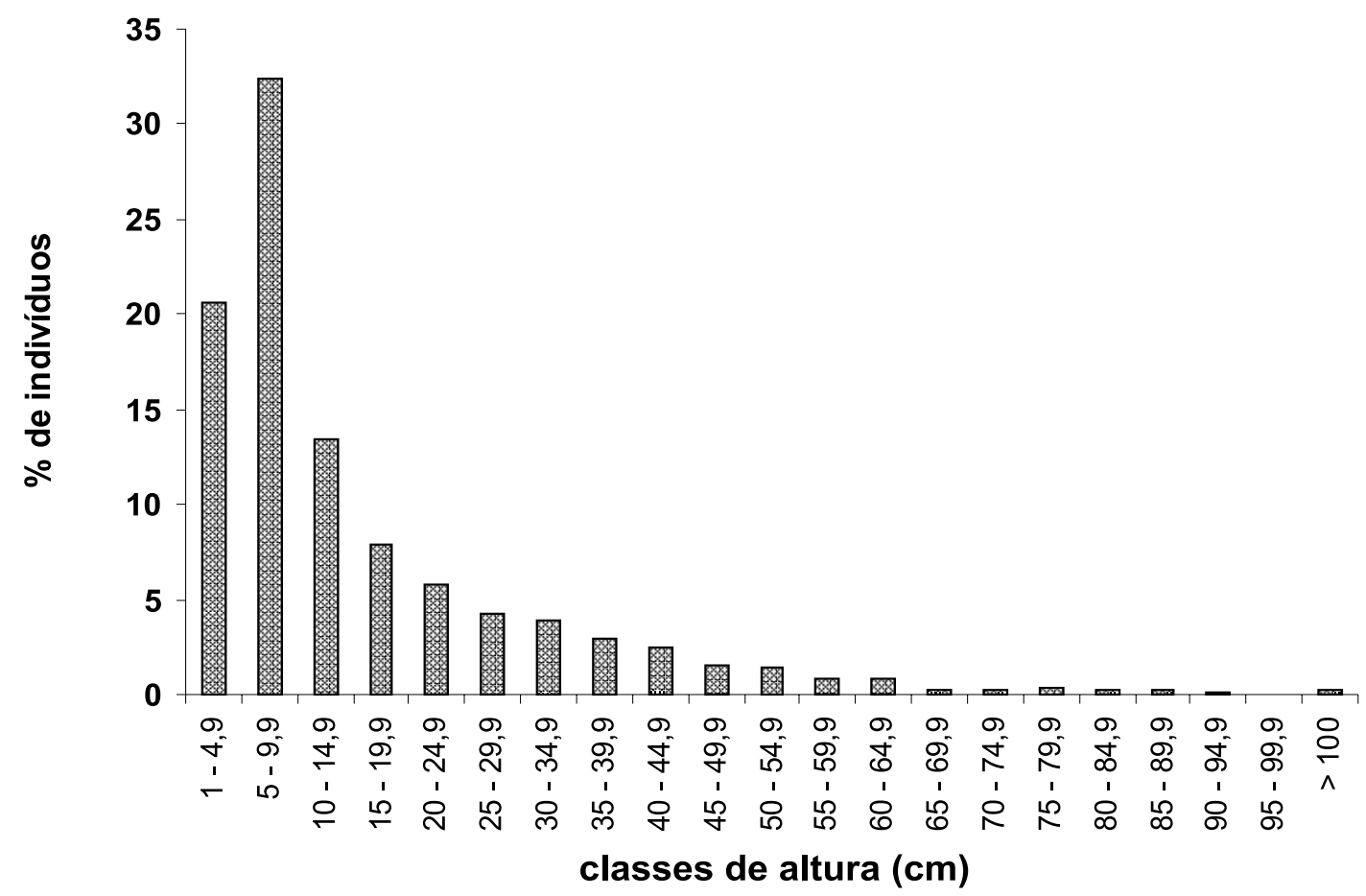

Figura 1 - Distribuição de freqüências de classes de altura dos indivíduos amostrados no levantamento fitossociológico em uma canga no Parque Estadual da Serra do Rola Moça, MG.

Figure 1 - Frequency distribution of height classes of the individuals sampled in the phyto-sociological survey in an area of ironstone outcrop, Serra do Rola Moça State Park, MG, Brazil. 
Quadro 2 - Parâmetros fitossociológicos das famílias amostradas em uma canga no PES Rola Moça, MG. Abreviaturas: ver Quadro 1

Table 2-Phyto-sociological parameters of the families sampled in an ironstone outcrop, Serra do Rola Moça State Park, MG, Brazil. See Table 1 for abbreviations

\begin{tabular}{lcccccc}
\hline Família & $\begin{array}{c}\mathrm{N}^{\circ} \\
\text { spp. }\end{array}$ & $\begin{array}{c}\text { FR } \\
(\%)\end{array}$ & $\begin{array}{c}\text { DR } \\
(\%)\end{array}$ & $\begin{array}{c}\text { DoR } \\
(\%)\end{array}$ & $\begin{array}{c}\text { IVC } \\
(\%)\end{array}$ & $\begin{array}{c}\text { IVI } \\
(\%)\end{array}$ \\
\hline Asteraceae & 7 & 12,83 & 8,69 & 31,35 & 20,02 & 17,63 \\
Poaceae & 3 & 12,39 & 23,06 & 12,01 & 17,53 & 15,82 \\
Orchidaceae & 3 & 11,06 & 26,50 & 6,37 & 16,43 & 14,64 \\
Cyperaceae & 2 & 10,18 & 19,62 & 5,05 & 12,33 & 11,62 \\
Euphorbiaceae & 2 & 11,50 & 7,16 & 7,60 & 7,38 & 8,76 \\
Verbenaceae & 1 & 7,96 & 3,02 & 13,00 & 8,01 & 8,00 \\
Velloziaceae & 2 & 8,41 & 7,25 & 7,91 & 7,58 & 7,86 \\
Melastomataceae & 2 & 6,19 & 1,16 & 5,29 & 3,23 & 4,22 \\
Loranthaceae & 1 & 2,21 & 0,23 & 8,08 & 4,16 & 3,51 \\
Fabaceae & 2 & 3,10 & 0,60 & 2,39 & 1,50 & 2,03 \\
Cactaceae & 1 & 4,42 & 0,65 & 0,22 & 0,43 & 1,76 \\
Portulacaceae & 1 & 3,54 & 0,65 & 0,01 & 0,33 & 1,40 \\
Apocynaceae & 2 & 2,65 & 0,74 & 0,21 & 0,47 & 1,20 \\
Lamiaceae & 1 & 1,77 & 0,19 & 0,32 & 0,25 & 0,76 \\
Rubiaceae & 1 & 1,33 & 0,37 & 0,06 & 0,21 & 0,59 \\
Malpighiaceae & 1 & 0,44 & 0,09 & 0,14 & 0,12 & 0,23 \\
\hline
\end{tabular}

Espécies com reprodução clonal corresponderam a $76 \%$ dos indivíduos, estando três entre as mais importantes, A. ingratus, B. fimbriata e $S$. caulescens. $A$. teres, uma orquídea importante em áreas de canga (VINCENT, 2004), participa da sucessão primária em afloramentos rochosos quartzíticos (ALVES e KOLBEK, 2000; NOGUEIRA et al., 2005). A associação com micorrizas é um fator imprescindível no ciclo de vida de certas orquídeas epilíticas, sendo alguns dos fungos simbiontes encontrados mesmo em áreas degradadas por mineração (NOGUEIRA et al., 2005). Plantas clonais também podem responder melhor no início da revegetação em locais restaurados, devido à conexão morfológica e fisiológica dos clones à planta-mãe e à alocação de recursos diferenciada entre as partes, o que permite melhor exploração do substrato (STUEFFER et al., 1996; IKEGAMI, et al., 2008). Considerando que freqüentemente ocorre diminuição na quantidade e variedade de polinizadores nas áreas degradadas por atividades de mineração devido às alterações no ambiente, essa estratégia permite que o indivíduo se espalhe, independentemente do sucesso da polinização para produzir sementes.

As gramíneas corresponderam a $23 \%$ do total de indivíduos amostrados, e têm características favoráveis para recuperação de áreas degradadas. Experimentos recentes com gramíneas nativas em áreas degradadas por mineração têm comprovado seu alto potencial para recuperação por fornecerem rápida cobertura do solo (MARTINS et al., 2001). Ainda, na colonização natural de rejeitos de mineração as espécies rizomatosas (entre elas, gramíneas do gênero Paspalum) contribuem para a estabilização dos taludes, ao crescerem e se espalharem pelo substrato (SHU et al., 2005). Neste estudo, $A$. ingratus, $B$. fimbriata e $P$. scalare, além de apresentar propagação vegetativa por rizomas, estão entre as espécies com maior VI, indicando um uso potencial na recuperação de áreas de mineração de ferro. De acordo com Martins et al. (2001), apesar da grande utilização de gramíneas exóticas invasoras como Melinis minutiflora e Brachiaria decumbens na cobertura de áreas degradadas, recentemente empresas de mineração passaram a se interessar por espécies nativas nos programas de recuperação. Essa substituição favorece a sucessão natural, uma vez que $M$. minutiflora tem alto poder competitivo em relação a espécies nativas (ÂNGELO et al., 2002).

A seleção de espécies para a recuperação de áreas degradadas pela extração de minérios deve levar em conta, ainda, a tolerância a altas concentrações de metais pesados no substrato. O grau de intemperização da rocha tem influência sobre os teores de metais pesados disponíveis, de forma que substratos nodulares podem ser mais tóxicos do que afloramentos rochosos (VINCENT e MEGURO, 2008), fato importante em projetos de recuperação sobre rejeitos de mineração. As espécies metalófilas podem ser usadas tanto para recuperar a cobertura vegetal quanto para fitorremediação em áreas contaminadas. Algumas espécies de Vellozia, as "canelasde-ema”, são consideradas metalófilas (ANTONOVICS et al., 1971; BROOKS, 1998). Esse gênero é importante em cangas por apresentar grande número de indivíduos, muitas vezes clonais, o que o torna adequado para projetos de recuperação. Estudos fisiológicos sobre as funções de plantas crescendo sobre canga como fitorremediadoras ainda são escassos. Entre os poucos publicados, uma pesquisa realizada em área de canga na Serra de Itabirito (QF) mostrou o acúmulo de metais pesados em folhas de Eremanthus erythropappus e E. glomerulatus (Asteraceae), Microlicia crenulata e Trembleya laniflora (Melastomataceae) (TEIXEIRA e LEMOS-FILHO, 1998), indicando tratar-se de espécies metalófitas. 
Apesar da ubiqüidade de diversas espécies, a heterogeneidade fisiográfica das cangas, resultando em alta diversidade, presença de endemismos e baixa similaridade florística entre áreas (JACOBI et al., 2007), e os altos teores de metais pesados no substrato (VINCENT, 2004) ratificam a importância de mais estudos ecológicos e geobotânicos pontuais de campos rupestres ferruginosos, para estabelecer procedimentos adequados de recuperação em cada área-alvo.

Iniciado em 2000, um grupo formado pelas maiores empresas de mineração do mundo, instituições de pesquisa e governos, organizou o projeto "Mineração, Minerais e Desenvolvimento Sustentável” (MMSD). Entre as recomendações do projeto estão o aumento do conhecimento e a utilização da biodiversidade de regiões metalíferas na restauração ambiental (WHITING et al., 2004). Estudos futuros que ampliem o conhecimento sobre os campos rupestres ferruginosos em Minas Gerais devem ser incentivados, pois estes estão distribuídos como manchas isoladas numa área de apenas $7.200 \mathrm{~km}^{2}$, em que ocorre intensa atividade mineradora. Ainda há grandes lacunas no conhecimento das comunidades vegetais e de serviços ecológicos com potencial de uso em áreas afetadas direta ou indiretamente pela extração de minério de ferro.

Lima et al. (2006) citaram a ausência do conhecimento prévio sobre as características dos ecossistemas como uma das principais causas da qualidade questionável de PRAD de minas no QF. Portanto, a caracterização da comunidade vegetal sobre canga e a discussão sobre a possibilidade de utilização de algumas espécies em programas de reabilitação ambiental são passos iniciais necessários, principalmente quando se observa o contexto geo-econômico do $\mathbf{Q F}$, com uma intensa atividade de mineração, gerando grande demanda por processos ambientais de licenciamento para pesquisa, concessões minerais e exploração.

\section{CONCLUSÕES}

A lista de espécies a serem priorizadas na definição de programas de recuperação de áreas degradadas por mineração de ferro deveria incluir: espécies provavelmente facilitadoras como Stachytarpheta glabra, Lychnophora pinaster e Mimosa calodendron; tolerantes a altas concentrações de metais pesados no substrato, como Vellozia spp.; espécies com crescimento clonal como gramíneas, ciperáceas e orquídeas epilíticas. Deve-se levar em conta, ainda, a posição hierárquica que as espécies ocupam na comunidade de campos ferruginosos, o que reforça a indicação de Andropogon ingratus, Bulbostylis fimbriata, Sophronitis caulescens e Lychnophora pinaster, além de Sebastiania glandulosa, que apresentaram os maiores valores de importância na comunidade e são freqüentes em outras áreas de canga do Quadrilátero Ferrífero. Deve-se procurar, quando possível, eleger adicionalmente outras espécies importantes em comunidades vizinhas à área que se pretende restaurar. Essa lista preliminar foi baseada em aspectos fitossociológicos, e recomenda-se a realização de novos levantamentos florísticos e fitossociológicos em campos ferruginosos do Quadrilátero Ferrífero, além de experimentos para avaliar o desempenho das espécies selecionadas, seja em viveiros, seja in situ. Recomenda-se, ainda, a utilização de espécies de poáceas, ciperáceas, asteráceas e orquidáceas com ocorrência em cangas, devido ao seu rápido crescimento, devendo-se testar a viabilidade da semeadura direta em áreas de reabilitação. No caso das orquídeas de canga, deve-se adotar a associação com micorrizas para aumentar a chance de sucesso no plantio.

\section{AGRADECIMENTOS}

À Fundação de Amparo à Pesquisa do Estado de Minas Gerais (FAPEMIG), pelo financiamento do projeto (CRA 89/03); ao Instituto Estadual de Florestas (IEF/ MG), pela licença concedida; a Aristônio Teles, Pedro L. Viana, Marcos Sobral e Rubens C. Mota, pela identificação das espécies; a José Eugênio do Carmo, Marina Beirão e Renzo A. Lanza, pelo auxílio no campo; e aos revisores anônimos, pela leitura crítica do manuscrito.

\section{REFERÊNCIAS}

AB'SABER, A. N. Os domínios morfoclimáticos na América do Sul. Geomorfologia, v.52, n.1, p.1-22, 1977.

ALVES, R. J. V. Morphological age determination and longevity in some Vellozia populations in Brazil. Folia Geobotanica Phytotaxa Praha, v.29, n.1, p.55-59, 1994. 
ALVES, R. J. V.; KOLBEK, J. Primary succession on quartzite cliffs in Minas Gerais, Brazil.

Biologia Bratislava, v.55, n.1, p.69-83, 2000.

ÂNGELO, J.G. M. et al. Diversidade vegetal em áreas de reabilitação de mineração de ferro, na mina de Alegria, em Mariana-MG. Revista Árvore, v.26, n.2, p.183-192, 2002.

ANTONOVICS, J.; BRADSHAW, A. D.; TURNER, R. G. Heavy metal tolerance in plants. Advances in Ecological Research, v.7, p.1-85, 1971.

ARAÚJO, F. S. et al. Estrutura da vegetação arbustivo-arbórea colonizadora de uma área degradada por mineração de caulim, em Brás Pires, MG. Revista Árvore, v.30, n.1, p.107-116, 2006.

BARTH, R. C. Avaliação da recuperação de áreas mineradas no Brasil. Viçosa, MG: SIF, 1989. 41p. (Boletim Técnico, 1)

BRADSHAW, A. Restoration of mined lands using natural processes. Ecological Engineering, v.8, n.4, p.255-269, 1997.

BROOKS, R. R. Geobotany and hyperaccumulators. In: BROOKS, R. R. (Ed.) Plants that hyperaccumulate heavy metals: Their role in phytoremediation, microbiology, archaeology, mineral exploration and phytomining. Cambridge: CAB International, 1998. p.55-94.

BRUNO, J. F.; STACHOWICZ, J. J.; BERTNESS, M. D. Inclusion of facilitation into ecological theory. Trends in Ecology and

Evolution, v.18, NUMERO, p.119-125, 2003.

BURKE, A. Inselbergs in a changing world global trends. Diversity and

Distributions, v.9, n.5, p.375-383, 2003.

CASTRO, J. et al. Use of shrubs as nurse plants: a new technique for reforestation in Mediterranean mountains. Restoration Ecology, v.10, n.2, p.297-305, 2002.

CONCEIÇÃO, A. A.; GIULIETTI, A. M.

Composição florística e aspectos estruturais de campo rupestre em dois platôs no Morro do Pai Inácio, Chapada Diamantina, Bahia, Brasil.

Hoehnea, v.29, n.1, p.37-48, 2002.

R. Árvore, Viçosa-MG, v.32, n.2, p.345-353, 2008
DEPARTAMENTO NACIONAL DE PRODUÇÃO MINERAL - DNPM. Sumário mineral.

Brasília: 2006. 122p.

DRUMMOND, G. M. et al. (Orgs.).

Biodiversidade em Minas Gerais - um atlas para sua conservação. 2.ed. Belo Horizonte: Fundação Biodiversitas, 2005. 222p.

GINOCCHIO, R.; BAKER, A. J. M. Metallophytes in Latin America: a remarkable biological and genetic resource scarcely known and studied in the region. Revista Chilena de Historia Natural, v.77, n.1, p.185-194, 2004.

GIULIETTI, A. M. et al. Flora da Serra do Cipó, Minas Gerais: caracterização e lista de espécies. Boletim de Botânica da Universidade de São Paulo, v.9, p.1-151, 1987.

IKEGAMI, M.; WHIGHAM, D.F.; WERGER, M.J.A. Optimal biomass allocation in heterogeneous environments in a clonal plant spatial division of labor. Ecological Modelling, v.213, n.2, p.156-164, 2008.

GRIFFITH, J. J.; TOY, T. J. Evolution in revegetation of iron-ore mines in Minas Gerais State, Brazil. Unasylva, v.52, n.1, p.9-15, 2001.

INSTITUTO BRASILEIRO DE MINERAÇÃO IBRAM. Contribuição do IBRAM para o zoneamento ecológico-econômico e o planejamento ambiental de municípios integrantes da APA-SUL RMBH. Brasília: 2003. 322p.

JACOBI, C. M. et al. Plant communities on ironstone outcrops - a diverse and endangered Brazilian ecosystem. Biodiversity and Conservation, v.16, n.7, p.2185-2200, 2007.

JACOBI, C. M.; CARMO, F. F. The Contribution of Ironstone Outcrops to Plant Diversity in the Iron Quadrangle, a Threatened Brazilian Landscape. AMBIO, v.37, n.4, p.324-326, 2008 a.

JACOBI, C. M.; CARMO, F. F. Diversidade dos campos rupestres ferruginosos no Quadrilátero Ferrífero, MG.

Megadiversidade, no prelo 2008b. 
LIMA, H. M.; FLORES, J. C. C.; COSTA, F. L. Plano de recuperação de áreas degradadas versus plano de fechamento de mina: um estudo comparativo. Revista Escola de Minas, v.59, n.4, p.397-402, 2006.

MARTINS, C. R.; LEITE, L. L.; HARIDASAN, M. Recuperação de uma área degradada pela mineração de cascalho com uso de gramíneas nativas.

Revista Árvore, v.25, n.2, p.157-166, 2001.

MEIRELLES, S. T.; PIVELLO, V. R.; JOLY, C. A. The vegetation of granite rock outcrops in Rio de Janeiro, Brazil, and the need for its protection. Environmental Conservation, v.26, n.1, p.10-20, 1999.

MUELLER-DOMBOIS, D.; ELLENBERG, H. Aims and methods of vegetation ecology. New York: Willey \& Sons, 1974. 547p.

NOGUEIRA, R. E. et al. Fungos micorrízicos associados a orquídeas em campos rupestres na região do Quadrilátero Ferrífero, MG, Brasil. Acta Botanica Brasilica, v. 19, n.3, p.417-424, 2005.

PADILLA, F. M.; PUGNAIRE, F. I. The role of nurse plants in the restoration of degraded environments. Frontiers in Ecology and the Environment, v.4, n.4, p.196-202, 2006.

PORTO, M. L.; SILVA, M. F. F. Tipos de vegetação metalófila em áreas da Serra dos Carajás e de Minas Gerais, Brasil. Acta Botanica Brasilica, v.3, n.1, p.13-21, 1989.

RIZZINI, C. T. Tratado de fitogeografia do Brasil. 2.ed. São Paulo: HUCITEC/ Universidade de São Paulo, 1997. 374p.

SIMMONS, G. C. Canga Caves in the Quadrilátero Ferrífero, Minas Gerais, Brazil. The National Speleological Society Bulletin, v.25, n.1, p.66-72, 1963.

SHU, W. S. et al. Natural colonization of plants on five lead/zinc mine tailings in southern China.

Restoration Ecology, v.13, n.1, p.49-60, 2005.
SPIER, C. A.; BARROS, S. M.; ROSIÈRE, C. A. Geology and geochemistry of the Águas Claras and Pico Iron Mines, Quadrilátero Ferrífero, Minas Gerais, Brazil. Mineralium Deposita, v. 38, n.6, p.751-774, 2003.

STUEFFER, J. F.; DE KROON, H.; DURING, H. J. Exploitation of environmental heterogeneity by spatial division of labor in a clonal plant.

Functional Ecology, v.10, n.3, p.328-334, 1996.

TEIXEIRA, W. A.; LEMOS FILHO, J. P. Fatores edáficos e a colonização de espécies lenhosas em uma cava de mineração de ferro em Itabirito, Minas Gerais. Revista Árvore, v.26, n.1, p.25-33, 2002.

TEIXEIRA, W. A.; LEMOS FILHO, J. P. Metais pesados em folhas de espécies lenhosas colonizadoras de uma área de mineração de ferro em Itabirito, Minas Gerais. Revista Árvore, v.22, n.3, p.381-387, 1998.

TOY, T. J.; GRIFFITH, J. J. Changing surface-mine reclamation practices in Minas Gerais, Brazil.

International Journal of Surfaces Mining, Reclamation and Environment, v.15, n.1, p.33-51, 2001.

TOY, T. J.; GRIFFITH, J. J.; RIBEIRO, C. A. A. Planejamento a longo prazo da revegetação para o fechamento de minas a céu aberto no Brasil. Revista Árvore, v.25, n.4, p.487-499, 2001.

VIANA, P. L.; LOMBARDI, J. Florística e caracterização dos campos rupestres sobre canga na Serra da Calçada, Minas Gerais, Brasil. Rodriguésia, v.58, n.1, p.159-177, 2007.

VINCENT, R. C. Florística, fitossociologia e relações entre a vegetação e o solo em áreas de campos ferruginosos no quadrilátero ferrífero, Minas Gerais. 2004. 145f. Tese (Doutorado em Ecologia) Universidade de São Paulo, São Paulo, 2004.

WHITING, S. N. et. al. Research priorities for conservation of metallophyte biodiversity and their potential for restoration and site remediation. Restoration Ecology, v.12, n.1, p.106-116, 2004. 
